\title{
CONAN - the cruncher of local exchange coefficients for strongly interacting confined systems in one dimension
}

\author{
N. J. S. Loft, ${ }^{1}$ L. B. Kristensen, ${ }^{1}$ A. E. Thomsen, ${ }^{1,2}$ A. G. Volosniev ${ }^{3,1}$ and N. T. Zinner ${ }^{1}$ \\ ${ }^{1}$ Department of Physics and Astronomy, Aarhus University, DK-8000 Aarhus C, Denmark \\ ${ }^{2} C P^{3}$-Origins 85 the Danish Institute for Advanced Study Danish IAS, \\ University of Southern Denmark, DK-5230 Odense M, Denmark. \\ ${ }^{3}$ Institut für Kernphysik, Technische Universität Darmstadt, 64289 Darmstadt, Germany
}

(Dated: October 15, 2018)

\begin{abstract}
We consider a one-dimensional system of particles with strong zero-range interactions. This system can be mapped onto a spin chain of the Heisenberg type with exchange coefficients that depend on the external trap. In this paper, we present an algorithm that can be used to compute these exchange coefficients. We introduce an open source code CONAN (Coefficients of One-dimensional N-Atom Networks) which is based on this algorithm. CONAN works with arbitrary external potentials and we have tested its reliability for system sizes up to around 35 particles. As illustrative examples, we consider a harmonic trap and a box trap with a superimposed asymmetric tilted potential. For these examples, the computation time typically scales with the number of particles as $O\left(N^{3.5 \pm 0.4}\right)$. Computation times are around 10 seconds for $N=10$ particles and less than 10 minutes for $N=20$ particles.
\end{abstract}

\section{INTRODUCTION}

The study of strongly interacting systems can be motivated by the realization that coherent quantum phenomena, such as high-temperature superconductivity, Helium superfluidity, and quantum magnetism, occur in systems where the particle interactions are intrinsically strong. In one-dimensional (1D) systems, examples are strong Coulomb interactions in quantum nanowires and in nanotubes [1], as well as linear compounds with strong exchange interactions that provide realizations of various spin chains [5]7]. To treat these 1D systems, some popular approaches include bosonization and Luttinger liquid theory [8], the Bethe ansatz [9], and, most recently, the density matrix renormalization group [10, 11] invented by Steven White.

It has now become possible to build setups with cold atomic gases that can provide quantum simulation of these low-dimensional quantum few- and many-body systems [12 16]. In particular, the Tonks-Girardeau gas [17, 18] consisting of strongly interacting bosons in $1 \mathrm{D}$ was realized in experiments 19 23. Luttinger-liquid behavior in interacting bosonic systems was also observed [24. More recently, strongly interacting fermionic systems were realized in 1D [25], also in the limit of just a few particles 26 29. In the latter setup it was shown that one can experimentally access strongly interacting few-body systems in the regime where they realize a Heisenberg spin chain [30. These developments were a motivation for our work during the last few years which resulted in the method presented below.

Before we proceed with our presentation, we overview some previous studies relevant to the present paper. In 1960 Girardeau [18] demonstrated the connection between strongly interacting bosons and spin-polarized/spinless fermions. For instance, it turns out that the ground state of a bosonic system can be obtained by taking the abso- lute value of the corresponding fermionic wave function. This connection was subsequently generalized in various ways. In 2004, within the context of atomic systems, Girardeau and Olshanii provided the so-called Fermi-Bose mapping strategy to describe two-component Fermi and Bose systems 31]. In 2006 Girardeau connected these observations to exchange interactions, thus, relating the mapping to spin models 32. In the strongly interacting regime a formal mapping that produces eigenstates of the total spin and the spin projection along one direction was proposed not long after [33, 34]. Note that this connection was also established within condensedmatter physics, where in 1990 Ogata and Shiba 35 used the Bethe ansatz solution to show how the 1D Hubbard model becomes a spin model for strong interactions. The spin models that were derived have exchange coefficients that are site-independent because the starting point was usually either homogeneous 1D space or a lattice model with some discrete translation invariance.

It was not until around 2013, when scientists realized that once you deviate from the homogeneous case, the spin models and particularly their exchange coefficients should be site-dependent, as they need to reflect the geometric landscape of the confinement potential. This was initially identified as a short-coming of the Fermi-Bose mapping in the case of harmonic confinement [36, 37. It was then realized that this does not spell the end of the Fermi-Bose mapping and effective spin model Hamiltonians 38 40, but it does mean that a different model that takes the external confinement into account should be used. This model implies that a confined $N$-body system has a set of distinct 'local exchange coefficients' that are completely specified by the external potential. The first reference to provide an explicit formula for these coefficients is Volosniev et al. [38. In this paper it was also realized that the exchange coefficients do not depend on the system composition (bosons/fermions/mixtures, two- or more internal degrees of freedom) as long as the interactions 
between all species are strong and the particle masses are the same. This paper was soon followed by several other papers exploring various approximations to these coefficients 41] and their applications 42,49.

Due to the presence of confinement in the relevant experimental setups, it is crucial to be able to calculate these coefficients. This is a complicated task, because the explicit formula for the local exchange coefficients involves computationally demanding $N-1$ dimensional integrals over an antisymmetrized $N$-body wave function (a Slater determinant) 38. In the present paper, we describe a procedure for computing these coefficients that bypasses the complexity of high-dimensional integrations, and introduce associated software called CONAN that computes the coefficients for up to 35 particles in arbitrary external potentials.

The source code to the CONAN program is freely available at [50] including pre-compiled versions that run out of the box. We ask that in any scientific publication based wholly or in part on CONAN, the use of CONAN must be acknowledged and the present paper must be cited.

The paper is organized as follows. In Sec. III we introduce the system and the spin chain Hamiltonian, $H_{s}$, that desribes it. In Sec. III we propose an algorithm for calculating parameters of $H_{s}$. In Sec. IV we start the presentation of CONAN by introducing the dimensionless variables that our software uses. A guideline of how to use CONAN can be found in Secs. V] and VI and in the documentation accompanying the program. Finally, in Sec. VII we give a brief overview of the present study.

\section{THE SYSTEM}

In this section we discuss the mapping of a strongly interacting one-dimensional gas onto a spin chain, see Fig. 1. A more detailed discussion of the mapping can be found in Ref. 40].

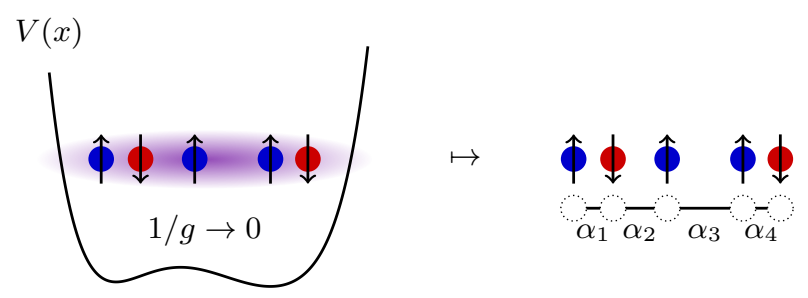

FIG. 1: Illustration of the mapping for a system with the interaction strength, $g$. In the strong interaction regime, $1 / g \rightarrow 0$, this system can be related (see the text) to a spin chain with trap-dependent exchange coefficients, $\alpha_{k}$.

\section{A. Confined strongly interacting gas}

We consider a system of particles in a one-dimensional trapping potential $V(x)$. All particles are of the same mass, $M$, and divided into two types which we call spin up and spin down. We denote by $N_{\uparrow}\left(N_{\downarrow}\right)$ the number of spin up (spin down) particles and by $N=N_{\uparrow}+N_{\downarrow}$ the total number of particles. The spin up particles have coordinates $x_{1}, x_{2}, \ldots, x_{N_{\uparrow}}$, whereas $x_{N_{\uparrow}+1}, \ldots, x_{N}$ are the positions of the spin down particles. We postulate that a spin up particle interacts with a spin down particle via a contact interaction of strenth $g>0$. Furthermore, we assume that other interactions have strength $\kappa g$ with $\kappa>0$. The dynamics of the system is governed by the following Hamiltonian

$$
\begin{aligned}
H= & \sum_{i=1}^{N} H_{0}\left(x_{i}\right)+g \sum_{i=1}^{N_{\uparrow}} \sum_{j=N_{\uparrow}+1}^{N} \delta\left(x_{i}-x_{j}\right) \\
& +\frac{\kappa g}{2} \sum_{i, j=1}^{N_{\uparrow}} \delta\left(x_{i}-x_{j}\right)+\frac{\kappa g}{2} \sum_{i, j=N_{\uparrow}+1}^{N} \delta\left(x_{i}-x_{j}\right),
\end{aligned}
$$

where the single-particle Hamiltonian is given by

$$
H_{0}(x)=-\frac{1}{2} \frac{\partial^{2}}{\partial x^{2}}+V(x),
$$

where for simplicity we put $\hbar=M=1$. We assume that particles of the same type are bosons. However, from the limit $\kappa \rightarrow \infty$ one can also learn about fermionic systems, see, e.g., Ref. [40.

\section{B. Effective spin chain model}

Before we consider the case of strong interaction, i.e., $1 / g \rightarrow 0$, we focus on the Tonks-Girardeau (TG) limit $(1 / g=0)$. In the TG limit, the wavefunctions vanish whenever any two particles meet, $x_{i}=x_{j}$. Therefore, in this limit the particles cannot exchange their positions, and for each ordering of particles (e.g., $x_{1}<x_{2}<\ldots<$ $x_{N}$ ) the system is described with a wave function of spinless fermions $\Phi_{0}\left(x_{1}, \ldots, x_{N}\right)$. For clarity from now on we assume that $\Phi_{0}$ corresponds to a ground state of spinless fermions. The discussion, however, can be extended easily to the excited manifolds. To construct $\Phi_{0}$ we find $\psi_{i}(x)$ - the real eigenstates of $H_{0}$, i.e.,

$$
H_{0}(x) \psi_{i}(x)=E_{i} \psi_{i}(x), \quad i=1,2, \ldots,
$$

where $E_{i}$ are the corresponding eigenvalues. From the $N$ lowest eigenstates, we construct the Slater determinant wavefunction, $\Phi_{0}$. The corresponding energy $E_{0}$ equals to the sum of the $N$ lowest single-particle energies, i.e., $E=$ $\sum_{i=1}^{N} E_{i}$. Note that the $N$-body system has $N ! /\left(N_{\uparrow} ! \cdot N_{\downarrow} !\right)$ distinct orderings, and therefore the ground state manifold of $H$ is $N ! /\left(N_{\uparrow} ! \cdot N_{\downarrow} !\right)$-fold degenerate.

When the interaction strength is moved slightly away from the TG limit, the wavefunctions become nonvanishing at $x_{i}=x_{j}$, thus allowing the particles to hop. To linear order in $1 / g$, we can describe this hopping using 
a spin chain Hamiltonian with nearest neighbor interaction:

$H_{s}=E_{0}-\sum_{k=1}^{N-1} \frac{\alpha_{k}}{g}\left[\frac{1}{2}\left(1-\boldsymbol{\sigma}^{k} \cdot \boldsymbol{\sigma}^{k+1}\right)+\frac{1}{\kappa}\left(1+\sigma_{z}^{k} \sigma_{z}^{k+1}\right)\right]$

where $\boldsymbol{\sigma}^{k}=\left(\sigma_{x}^{k}, \sigma_{y}^{k}, \sigma_{z}^{k}\right)$ are the Pauli matrices acting on the spin of the particle at the $k$ th position and $\alpha_{k}$ are the exchange coefficients. In Ref. [40] it was shown that

$$
\alpha_{k}=\frac{\int_{x_{1}<x_{2}<\cdots<x_{N-1}} \mathrm{~d} x_{1} \ldots \mathrm{d} x_{N-1}\left|\frac{\partial \Phi_{0}}{\partial x_{N}}\right|_{x_{N}=x_{k}}^{2} .}{\int_{x_{1}<x_{2}<\cdots<x_{N}} \mathrm{~d} x_{1} \mathrm{~d} x_{2} \ldots \mathrm{d} x_{N}\left|\Phi_{0}\right|^{2}} .
$$

For convenience we assume that $\Phi_{0}$ is normalized such that $\int_{x_{1}<x_{2}<\cdots<x_{N}} \mathrm{~d} x_{1} \mathrm{~d} x_{2} \ldots \mathrm{d} x_{N}\left|\Phi_{0}\right|^{2}=1$. The exchange coefficients $\alpha_{k}$ are called geometric coefficients, derived from the fact that they only depend on the geometry of the trap potential.

\section{ALGORITHM FOR CALCULATING $\alpha_{k}$}

Access to the coefficients $\alpha_{k}$ would effectively solve the strongly interacting $N$-body trapped system, and, thus, we seek a way to compute them. In principle, one could find the $N$ lowest energy single-particle wavefunctions $\psi_{i}$, construct the Slater determinant wavefunction, $\Phi_{0}$, and evaluate the $(N-1)$-dimensional integral in Eq. (5). However, multidimensional integrals are computationally demanding. Therefore, for more than just a few particles, we need to cast $\alpha_{k}$ in a form better suited for numerical calculations. In Appendix $\mathrm{A}$ we show that $\alpha_{k}$ can be expressed as a sum of one-dimensional integrals

$$
\begin{aligned}
\alpha_{k}= & 2 \sum_{i=1}^{N} \sum_{j=1}^{N} \sum_{l=0}^{N-1-k} \frac{(-1)^{i+j+N-k}}{l !}\left(\begin{array}{c}
N-l-2 \\
k-1
\end{array}\right) \\
& \times \int_{a}^{b} \mathrm{~d} x \frac{2 m}{\hbar^{2}}\left(V(x)-E_{i}\right) \psi_{i}(x) \frac{\mathrm{d} \psi_{j}}{\mathrm{~d} x} \\
& \times\left[\frac{\partial^{l}}{\partial \lambda^{l}} \operatorname{det}\left[(B(x)-\lambda \mathbf{I})^{(i j)}\right]\right]_{\lambda=0} \\
& +\sum_{i=1}^{N}\left[\frac{\mathrm{d} \psi_{i}}{\mathrm{~d} x}\right]_{x=b}^{2}
\end{aligned}
$$

where I denotes the identity matrix, ( $)^{(i j)}$ denotes the $i j$ 'th submatrix obtained by removing the $i$ 'th column and the $j$ 'th row, $B(x)$ is a $N \times N$ symmetric matrix with the $m n$ 'th entry defined as the partial overlap of $\psi_{m}$ and $\psi_{n}$, i.e.,

$$
[B(x)]_{m, n}=\int_{a}^{x} \mathrm{~d} y \psi_{m}(y) \psi_{n}(y) .
$$

The interval $[a, b]$ denotes the support of $V(x)$. For example, for a harmonic oscillator potential the support is the entire $x$-axis, i.e., $[a, b]=(-\infty, \infty)$; for a hard box, defined from $x=0$ to $x=L$, we have $[a, b]=[0, L]$.

In Eq. (6) the $(N-1)$-dimensional integral has been rewritten as a sum of terms which scale more advantageously with $N$. We note that similar reductions have been discussed in relation to calculating the densities of strongly interacting systems, see Ref. 33. Unfortunately, the new expression contains the l'th order derivative in the square brackets which complicates numerical calculations as $N$ increases. Therefore, we would like to simplify the expression further.

\section{A. Simplifying the determinant}

From the standpoint of an effective numerical implementation of Eq. (6) the complicated part is the evaluation of the derivatives of the determinant,

$$
\left[\frac{\partial^{l}}{\partial \lambda^{l}} \operatorname{det}\left[(B(x)-\lambda \mathbf{I})^{(i j)}\right]\right]_{\lambda=0} .
$$

Our method for evaluating this expression is due to the fact that $B$ is symmetric (and real because we have chosen real wavefunctions $\psi_{i}$ ), and, hence, diagonalizable using an orthogonal matrix $U=\left(\mathbf{u}_{1} \ldots \mathbf{u}_{N}\right)$ such that $B=$ $U^{\mathrm{T}} D U$, where $D$ is a diagonal matrix composed of the eigenvalues of $B$. We note that taking the $i j$ 'th submatrix of $B$ is equivalent to removing a row and a column from $U^{\mathrm{T}}$ and $U$ respectively. This observation allows us to show (see Appendix B for details) that the expression in Eq. (8) can be written as

$$
(-1)^{i+j} l ! \mathbf{u}_{j}^{\mathrm{T}}\left(\sum_{n=0}^{l} p_{l-n} D^{-(n+1)}\right) \mathbf{u}_{i},
$$

where $p_{k}$ are the coefficients of the polynomial

$$
\operatorname{det}(D-\lambda \mathbf{I})=p_{N} \lambda^{N}+\ldots+p_{1} \lambda+p_{0} .
$$

Several comments are in order here. First of all, as $D$ is diagonal, it can be easily inverted as long as its entries are nonzero. In Appendix $\mathrm{B}$ we prove that this is in fact the case. Secondly, the coefficients $p_{k}$ are easily computable because $\operatorname{det}(D-\lambda \mathbf{I})$ is a determinant of a diagonal matrix. Thirdly, a further reduction in computation requirements can be achieved by doing the sum over $l$ inside the integral. Then we diagonalize $B$ only once, rather than once for each $l$. Therefore, to evaluate the integrand we need to compute the expression

$$
\begin{aligned}
& (-1)^{i+j} \sum_{l=0}^{N-1-k}\left(\begin{array}{c}
N-2-l \\
k-1
\end{array}\right) \frac{1}{l !}\left[\frac{\partial^{l}}{\partial \lambda^{l}} \operatorname{det}\left[(B-\lambda \mathbf{I})^{(i j)}\right]\right]_{\lambda=0} \\
& =\mathbf{u}_{j}^{\mathrm{T}}\left[\sum_{l=0}^{N-1-k}\left(\begin{array}{c}
N-2-l \\
k-1
\end{array}\right) \sum_{n=0}^{l} p_{l-n} D^{-(n+1)}\right] \mathbf{u}_{i} \cdot(11)
\end{aligned}
$$

Finally, and perhaps the most interestingly, the expression in Eq. (11) depends on $i$ and $j$ only through $\mathbf{u}_{i}$ and 
$\mathbf{u}_{j}$. Hence, the vast majority of the computations are independent on $i$ and $j$ which prompts us to take the sum over $i$ and $j$ inside the integral in Eq. (6) as well, and reuse the result for the derivatives of the determinant. The procedure reduces the computation time as the derivatives have to be computed only once for each $x$ rather than computing it $\sim N^{2}$ times.

\section{B. Procedure}

Here we outline our method for computing the coefficients $\alpha_{k}$ from Eq. (6), where we first take all the sums for a given $x$ and then perform the integration. To take the sums the following is done:

1. The entries of $B(x)$ are evaluated.

2. The matrix $B(x)$ is diagonalized.

3. The coefficients $p_{k}$ are computed.

4. The matrices $D^{-(n+1)}$ are computed for all $0 \leq n \leq$ $N-1-k$ utilizing that $D$ is diagonal.

5. The (diagonal) matrix inside the square brackets of Eq. [11 is evaluated.

6. The sum over $i$ and $j$ is taken. For this the product in Eq. (11) is multiplied by the appropriate factors of $\psi_{i}, \mathrm{~d} \psi_{j} / \mathrm{d} x$, and $\left(V-E_{i}\right)$.

From the procedure sketched above we can estimate how the computation time scales with the number of particles, $N$. It seems that the steps 2,5 , and 6 are the most demanding for large $N$, because the computation time of all these steps scales roughly as $O\left(N^{3}\right)$. Still, this scaling is surprisingly good.

To further reduce the computation time we have exploited the fact that modern computers have multiple computing cores. The easiest way to go about parallelizing the program is to let each core compute a separate geometric coefficient. In our implementation there is not a great overlap in computation different coefficients, therefore, this way of parallelizing leads to a much more effective use of the computing power. Furthermore, the geometric coefficients only have a use when all of them are known, so this parallelizing cannot slow down the process.

\section{Arbitrary precision matrix computations}

There is one caveat associated with the outlined procedure which we should like to bring attention to. The success of our algorithm relies on an accurate diagonalization of $B(x)$, which is composed of partial overlap integrals of the wave functions. Some of these overlaps may be much smaller than the others depending on which overlap and to what value of $x$ it is being evaluated. Therefore, the entries of $B$ might span many orders of magnitude, and the use of too small precision in the diagonalization of $B$ might yield a wrong outcome.

It turned out that the usual machine precision is insufficient for $N \sim 7$. This is likely due to an enhanced error because the eigenvalues are taken to a high negative power in Eq. 11. We have found ourselves forced to implement a diagonalization routine using numbers with until several thousands bits precision (for large $N$ ) in order to determine the eigenvalues and eigenvectors accurately. The requirement for the numerical precision to this diagonalization scales with the number of particles so this inevitably influences the original estimate of an $O\left(N^{3}\right)$ computation time. In Section $\mathrm{VI}$ we will see that the computation time typically scales with $O\left(N^{3.5 \pm 0.4}\right)$.

\section{Computation of $B(x)$}

The computation of $B(x)$ from Eq. (7) is straightforward. It requires only the eigenstates of the one-particle Hamiltonian $H_{0}(x)$ from Eq. (2), and a numerical procedure to perform the integration in Eq. (7). Both of these requirements can be fulfilled by picking a suitable basis of states for the one-body problem. As such a basis, we choose the eigenstates of a hard box potential on the interval $[0, L]$, i.e.,

$$
\phi_{n}(x)=\sqrt{\frac{2}{L}} \sin \left(\frac{n \pi x}{L}\right), \quad n=1, \ldots, N_{b},
$$

where $N_{b}$ defines the basis size used. The basis of $N_{b}$ box wavefunctions in Eq. 12 form an orthogonal basis on $[0, L]$, which is complete in the limit $N_{b} \rightarrow \infty$.

To solve the one-body problem accurately we need to pick the values for $N_{b}$ and $L$. The number of basis states should be chosen much larger than the number of particles, $N_{b} \gg N$, such that the higher momentum contributions are not important. For instance, for the systems from Sec. VI correct results can be obtained using a basis with around a few hundred elements.

The basis states are defined on a finite interval, therefore, $L$ should be chosen sufficiently large such that the wavefunctions, $\psi_{i}$, are effectively confined to the region $[0, L]$. In other words, putting $a=0$ and $b=L$ in Eq. (6) should not change the value of $\alpha_{k}$ within a given precision.

After expressing the single-particle Hamiltonian, $H_{0}(x)$, as a $N_{b} \times N_{b}$ matrix in the basis of box eigenstates, we find the expansion coefficients for the wavefunctions, $\psi_{i}$, through the usual diagonalization procedure. Let us denote with

$$
[C]_{i, m}=\int_{0}^{L} \mathrm{~d} x \phi_{m}(x) \psi_{i}(x),
$$

the expansion coefficients, and with $C$ the $N \times N_{b}$ matrix of these. Then we derive the simple expression (see Appendix CD for the matrix $B$

$$
B(x)=C f(x) C^{\mathrm{T}},
$$


where $f(x)$ is the matrix:

$$
[f(x)]_{m, n}=\left\{\begin{array}{cl}
\frac{1}{\pi}\left[\frac{1}{m-n} \sin \left((m-n) \frac{\pi x}{L}\right)\right. & \\
\left.-\frac{1}{m+n} \sin \left((m+n) \frac{\pi x}{L}\right)\right] & \text { for } m \neq n \\
\frac{1}{\pi}\left[\frac{\pi x}{L}-\frac{1}{2 m} \sin \left(\frac{2 m \pi x}{L}\right)\right] & \text { for } m=n
\end{array}\right.
$$

The elements of this matrix are less computationally expensive to calculate than the original integrals in the $B(x)$-matrix, and the coefficient matrix $C$ only needs to be calculated once. Calculating $B(x)$ by using Eq. (14) is therefore significantly faster than performing the $N^{2}$ integrals in Eq. 7) numerically. It should be noted that this increase in speed does not alter the way the computation time scales with the number of particles. Simply doing the $N^{2}$ integrals from Eq. (7) would ideally scale as $O\left(N^{2}\right)$, but in practice increases a bit faster due to the difficulty of integrating the rapidly oscillating excited states that come into play when $N$ becomes large. Similarly, calculating $B(x)$ using Eq. (14) requires 2 matrix products involving the $C$-matrix. This matrix has $N$ rows and $N_{b}$ columns, and so the calculation time scales as $O\left(N^{2} N_{b}^{2}\right)$. Because the basis size does not need to be altered significantly as $N$ is increased, the scaling for this calculation is also close to $O\left(N^{2}\right)$.

\section{UNITS AND CHANGE OF UNITS}

Using the algorithm presented in the previous section we wrote a code CONAN, which produces $\alpha_{k}$ for given $V(x)$ and $N$. We start the presentation of our code by describing the conversion of the relevant quantities into unitless numbers that CONAN works with.

When looking at the expressions above, only two types of dimensions appear: dimensions of length and dimensions of energy. Therefore, we need to specify what units are used for lengths and energies. We let $\ell$ to be some unit of length, and define from this the unit of energy as

$$
\varepsilon=\frac{1}{2 \ell^{2}} .
$$

Let the unitless variable that corresponds to a quantity $q$ be denoted as $\tilde{q}$, i.e., $\tilde{x}=x / \ell$ and $\tilde{V}(x)=V(x) / \varepsilon$. CONAN assumes that $\tilde{x}, \tilde{V}$ variables are used in Eq. (2), and produces the corresponding $\tilde{\alpha}_{k}$. To convert $\tilde{\alpha}_{k}$ to $\alpha_{k}$ one should use the equation

$$
\tilde{\alpha}_{k}=\ell^{3} \alpha_{k},
$$

which follows directly from our definition of dimensionless quantities.

The procedure for translating from a physical system to the language of the program and back works as follows:

1. Pick a unit of length $\ell$.

2. Pick a sufficiently large box of length $\tilde{L}$.
3. Find the dimensionless potential as $\tilde{V}(\tilde{x})=$ $V(x / l) / \varepsilon$.

4. Enter $\tilde{L}$ and $\tilde{V}(\tilde{x})$ into the program and run it.

5. Convert $\tilde{\alpha}_{k}$ to $\alpha_{k}$ using Eq. (17).

As an example, let us consider a simple harmonic oscillator,

$$
V(x)=\frac{\omega^{2} x^{2}}{2} .
$$

For the concrete implementation in the program, we should shift it $x \rightarrow x-L / 2$ such that the minimum of $V(x)$ is in the center of the box, but this matters not for the present discussion.

A reasonable choice for the unit of length and the corresponding unit of energy is

$$
\ell=\sqrt{\frac{1}{\omega}}, \quad \varepsilon=\frac{1}{2} \omega .
$$

With these definitions, the numerical potential to feed into the program becomes

$$
\tilde{V}(\tilde{x}) \equiv \frac{1}{\varepsilon} V(\tilde{x} \ell)=\tilde{x}^{2},
$$

and when the program finishes calculating the coefficients $\tilde{\alpha}_{k}$, these should be converted back to dimensionful quantities as

$$
\alpha_{k}=\omega^{\frac{3}{2}} \tilde{\alpha}_{k}
$$

With this example in mind, we can now approach the subject of how the program behaves when we rescale the units. Assume we rescale the unit of length by a factor $\delta$. Because the unit of energy is connected to $\ell$ by Eq. (16), this would also rescale the unit of energy. The scaling then is

$$
\ell \rightarrow \delta \ell, \quad \varepsilon \rightarrow \delta^{-2} \varepsilon, \quad \tilde{V}(\tilde{x}) \rightarrow \delta^{2} \tilde{V}(\delta \tilde{x})
$$

Similarly, a rescaling of the energy by a factor $\lambda$ implies a rescaling of the unit of length,

$$
\varepsilon \rightarrow \lambda \varepsilon, \quad \ell \rightarrow \lambda^{-\frac{1}{2}} \ell, \quad \tilde{V}(\tilde{x}) \rightarrow \lambda^{-1} \tilde{V}\left(\lambda^{-\frac{1}{2}} \tilde{x}\right)
$$

Note that these two types of scaling are identical up to a change of notation if $\delta=\lambda^{-2}$.

For the harmonic oscillator in the example above, we see that changing the unit of energy to $\gamma \omega / 2$ yields the following change of the unitless potential

$$
\tilde{V}(\tilde{x}) \rightarrow \gamma^{-1} \tilde{V}\left(\gamma^{-\frac{1}{2}} \tilde{x}\right)=\gamma^{-2} \tilde{x}^{2}
$$

The change of the units can be seen as the change of the size of the potential. For some potentials, this observation might be used to yield scaling laws for $\alpha_{k}$, as explored in the following section. 


\section{A. Scaling of the coefficients with potential strength}

As mentioned above, the understanding of how the units enter in the problem allows for the derivation of scaling laws for $\alpha_{k}$. To see this, note first that besides the number of particles, the only information about the system entered into the calculations is the potential $\tilde{V}(\tilde{x})$. In other words, if we pick a sufficiently large $\tilde{L}$ (and specify other precision-parameters, as described in Section V], the coefficients should depend only on the potential, i.e.,

$$
\tilde{\alpha}_{k}=\tilde{\alpha}_{k}[\tilde{V}(\tilde{x})] .
$$

Assume now that we are dealing with a potential which is a homogeneous function, that is, a function for which there exists a point $a \in(0, L)$ and a real number $s$, such that for any $k \in \mathbb{R}$ the following holds:

$$
V(k(x-a))=k^{s} V(x-a) .
$$

One example of such a potential is the harmonic oscillator $(s=2)$ introduced in the previous section.

For potentials of this type, a scaling of the size of the potential may be countered by a rescaling of $\ell$, so that the combinations of the two scalings keep $\tilde{V}$ and, therefore, $\tilde{\alpha}$ unchanged. As shown in Appendix D this leads to the following scaling-law when the potential is scaled by a factor $\gamma$,

$$
\alpha_{k}[\gamma V]=\gamma^{\frac{3}{s+2}} \alpha_{k}[V] .
$$

For the harmonic case with $s=2$ the data from the program, run with varying potential-sizes, confirms that the coefficients scale as $\gamma^{\frac{3}{4}}$.

\section{GUIDE TO THE USE OF CONAN}

For a successful use of CONAN one has to understand in detail the input parameters given to the program. In this section we list these parameters, and discuss the means to estimate accuracy of results. To illustrate our code, in Section VI we consider two specific examples: a harmonic potential and an asymmetric tilted potential in a box.

1. The number of particles, $N$. Note that errors on some of the coefficients might become upredictable and large even for simple potentials, therefore, it is not advised to use $N>35$. The option $-\mathrm{N}$ specifies the number of particles.

2. The smooth dimensionless trapping potential, $\tilde{V}(\tilde{x})$. This potential should trap the system on the interval $[0, \tilde{L}]$, i.e., the potential at the boundaries ( 0 or $\tilde{L}$ ) of the box, that we use to expand the one-body wave functions, should be much larger than the typical energy scale. In other words the $N$ lowest-energy single-particle wavefunctions should be 'essentially' located in the interval $[0, \tilde{L}]$. The option $-V$ specifies the dimensionless potential as a mathematical function. The length of the box may be changed from the default value, $\tilde{L}=100$, by submitting another value using the option $-\mathrm{L}$.

Note that a box trap should be chosen to be zero, i.e., $\tilde{V}(\tilde{x})=0$. This choise is required due to the numerical integration routines, that are used to calculate the matrix elements for the potential.

3. The number of basis states, $N_{b}$. This number should be chosen such that the $N$ lowest-energy singleparticle wavefunctions are accurately represented in the basis of box states. The default value of the basis size in CONAN is $N_{b}=300$. The option -b specifies this number to a different value.

One can check whether $N_{b}$ is sufficiently large by comparing the results for different values of $N_{b}$, for instance $N_{b}=200,400,600,800 ; N_{b}$ should be chosen in the range where the coefficients do not change with changing $N_{b}$ (within some desired precision). Typically it is not a problem to choose $N_{b}$ larger than necessary. However, very large $N_{b}$ might lead to instabilities in the numerical calculations of the potential in the basis of box states, $V_{n m}=\int_{0}^{L} \mathrm{~d} x \phi_{n}(x) \phi_{m}(x) V(x)$. These instabilities are due to quickly oscillating integrands in the matrix elements and can be avoided by adapting the intergration routines.

Increasing the basis size increases the computation time through the diagonalization of the $N_{b} \times N_{b}$ Hamiltonian matrix, but this increase is almost independent on $N$. So if one is performing a few calculations for a large $N \sim 30$ system, where the computation time is an hour or so, it is a good idea to pick $N_{b}$ larger than necessary. On the other hand, if one is performing many calculations for small $N \sim 10$ with almost identical potentials (say, for noise studies as in Ref. [51), then the computation time can be decreased by choosing the minimal $N_{b}$ that yields the desired accuracy.

4. The bit precision on the arbitrary precision calculations, $p$. The default value of $p$ is 256 . If the bit precision is not sufficiently large, CONAN will run into an error or give coefficients which are several orders of magnitude different from each other. An accurate result is then obtained by increasing the value of $p$ using the option -p. As a rule of thumb, always choose the bit precision as a power of 2 , i.e., it should be increased in steps $p=256,512,1024$, 2048. The computation time depends strongly on the bit precision (see the next section for an illustration), therefore, one does not want to set $p$ higher than necessary. For just a few particles, $N \lesssim 5$, it suffices to use a bit precision smaller than the default value, say $p=64$ or $p=128$. 
While working with CONAN we noticed, that if a reasonable result can be obtained with the chosen bit precision, then typically this result is accurate. However, the accuracy can be confirmed only by increasing $p$.

5. The absolute or relative precision on the calculation of the single-particle wavefunctions and energies. These are changed using the options--abs-solver and --rel-solver respectively. Other input parameters are the absolute or relative precision on the calculation of the integral in Eq. (6) (changed using--abs-final and--rel-final). The default values of these parameters lead to accurate results for $N<30$ for potentials similar to the presented in the next section.

The above list serves as a checklist one should review before submitting an input to CONAN. There are other settings which can be changed in order to obtain a specific output format or for parameter dependent potentials, please consult the documentation for further details.

\section{A. On accuracy of results}

Even if CONAN does not return an error, the resulting coefficients may be wrong. Therefore, it is important to evaluate the result from the program. First of all, one should ask oneself whether it makes sense to interpret the coefficients as local exchange coefficients, provided the trapping potential. Typically, the coefficients will resemble the inverted potential $-V$, see for instance the examples in Sec. VI or Ref. [51. Next one should run CONAN again with an increased basis size and/or precision, and compare the results. One should keep increasing the basis size and/or precision until every coefficient is determined to the desired degree of precision.

For $N>30$, we advise the user to be extra cautious because the complexity of the calculations introduce errors in the results that become harder and harder to avoid, even with an increased basis size and precision parameters. Because the calculation time is also large, it becomes very tedious to estimate the precision of the result by peforming multiple calculations with different precision settings. There is, however, a way to estimate the error on the coefficients that works also for large values of $N$ : We know that reflecting the potential around its center point also reflects the coefficients, i.e., $V(x) \mapsto V(L-x)$ yields $\alpha_{k} \mapsto \alpha_{N-k}$. Thus comparing $\alpha_{k}$ calculated for $V(x)$ and $\alpha_{N-k}$ for $V(L-x)$ digit by digit, we can estimate the error on the coefficients. This method works because the coefficients we compare are calculated differently, i.e., we do not compare the results of identical numerical routines. In the special case of a symmetric potential, i.e., $V(x)=V(L-x)$, we can compare the coefficients directly by checking to which degree of accuracy the coefficients are symmetric, i.e., $\alpha_{N-k}=\alpha_{k}$.
We are hesitante to state something more specific about the precision of the results, because every case should be considered separately. However, we do note a few trends. For $N \leq 25$ high precision is only a matter of picking $N_{b}$ and $p$ sufficiently large, then the error can be reduced to less than $0.0001 \%$ or $0.00001 \%$. Increasing the number of particles to $N \approx 30$ introduces a small error on a few coefficients of the order $0.001 \%$ that cannot be easily reduced by changing $N_{b}$ or the precision parameters. We believe, these errors arise due to numerical instabilities, and we see no way to decrease them in the current version of the code. We also note that the errors are not evenly distributed among the $N-1$ coefficients: a few coefficients gain larger errors while the remaining coefficients seem accurate. This trend of non-uniformly distributed errors continues as $N$ increases. For $N=35$ the largest errors are of the order $0.1 \%$ to $1 \%$, depending on the system. For $N$ around 40 the errors become unacceptably large, but because the computation time is several hours for such large $N$, we have not done any systematic studies in this range of $N$. Therefore, we recommend to run CONAN for at most $N \approx 35$.

\section{EXAMPLES}

Let us turn to two specific examples that illustrate the program. In these examples we calculate the geometric coefficients for various values of $N$. As $N$ increases we gradually increase the basis size $N_{b}$ and bit precision $p$ in an effort to obtain high precision results while keeping the computation time low. We run CONAN on a computer with an Intel Xenon processor (CPU E5-2630 v3 @ 2.40 $\mathrm{GHz} \times 8)$ and note the computation time, $T$. To get an estimate of how $T$ scales with $N$, we fit our data to the model $T=a+b \cdot N^{c}$, where $a, b, c$ are fit parameters. We find that the computation time typically scales as $O\left(N^{3.5 \pm 0.4}\right)$ (depending on the potential and range of $N$ values). We also find that a calculation for $N=10$ particles takes approximately 10 seconds, and computations for $N=20$ can be done in less than 10 minutes.

\section{A. Harmonic potential}

Let us first calculate the geometric coefficients for a harmonic oscillator potential

$$
V(x)=\frac{1}{2} \omega^{2} x^{2},
$$

using the typical oscillator units given in Eqs. (19). Note that these coefficients for $N \leq 30$ were already presented in Ref. 47. Using the oscillator units introduced in Section [IV] we write the dimensionless potential

$$
\tilde{V}(\tilde{x})=\tilde{x}^{2} .
$$

However, we cannot submit the above potential to CONAN because the potential has its minimum at the bound- 

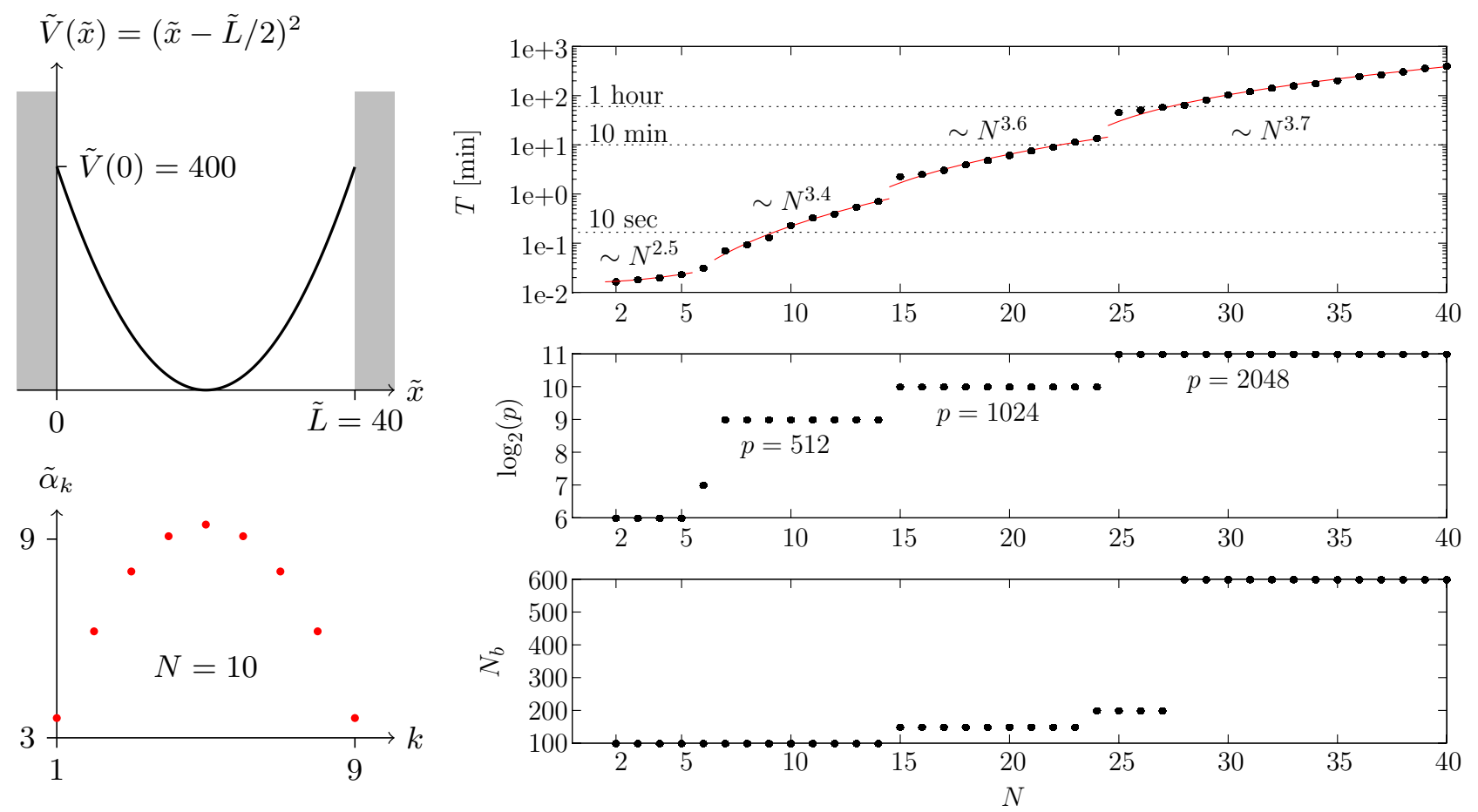

FIG. 2: Harmonic oscillator. To the left we sketch the harmonic potential submitted to CONAN and the calculated geometric coefficients for $N=10$. To the right, on the top panel, we plot the computation time $T$ versus the number of particles $N$ including the scaling for a fixed $p$. Below we show the chosen bit precision $p$ (middle) and basis size $N_{b}$ (bottom). The errors on the calculated coefficients are below $0.0001 \%$ for $N<29$ which increases to $\sim 0.1 \%$ for $N=35$ and becomes even larger for larger $N$, see the main text for further discussion.

ary of the box, so we shift the minimum of the potential to the center of the box,

$$
\tilde{V}(\tilde{x})=(\tilde{x}-\tilde{L} / 2)^{2} .
$$

At the boundaries of the box, the above potential yields $\tilde{V}(0)=\tilde{V}(\tilde{L})=\tilde{L}^{2} / 4$, so picking $\tilde{L}=40$ leads to the value of the potential at the boundary being 400 . This number is much larger than any other energy scale in the problem, which is what we want.

We calculate the geometric coefficients for $N=2, \ldots, 40$ with gradually increasing basis size $N_{b}$ and bit precision $p$. An input line to the program may look like

$$
\begin{aligned}
& \text {./Conan -V '(x-L/2) ^2' -L } 40 \text {-N } 8 \text {-p } 512 \text {-b } \\
& 100
\end{aligned}
$$

The basis size, bit precision and computation time for $N=2, \ldots, 40$ are shown in Figure 2. In the top panel of the figure we show the computation time $T$ versus $N$. We see that the computation time increases noticably when $p$ is increased. For every plateaux characterized with $p=64,512,1024$ and 2048, we calculate how the computation time scales with $N$ and see that it typically scales with $O\left(N^{3.5 \pm 0.2}\right)$.

The accuracy of these results can be estimated from the calculations with a larger number of basis states and higher precision parameters up to around 30 particles. Because the potential is symmetric around the middle of the box, we may also estimate the precision by checking to which degree the coefficients are symmetric, $\alpha_{k} \stackrel{?}{=} \alpha_{N-k}$.

For $N<29$ the estimated in this way error on the coefficients is less than $0.0001 \%$. For $N=30$ the error on a few coefficients increases to $0.001 \%$, while most of the coefficients are symmetric to a higher degree of precision. For $N=33$ the error on the most imprecise coefficients is $\sim 0.01 \%$, growing to $\sim 0.1 \%$ for $N=35$. It is worth noticing that the precision on the $N=35$ result is not increased by increasing the basis size to $N_{b}=$ 800 and the absolute and relative integral precision to 1e-08 using the options --abs-final and --rel-final, indicating that the precision cannot become better for these large $N$ results in the current version of CONAN due to numerical instabilities as discussed in the final paragraph of SectionVA. Pushing the limits of CONAN's capabilities by increasing $N$ further, we note that the error increases to a few percent for $N=37$ and up to the worst case deviations $\sim 100 \%$ for $N=40$. Clearly, one cannot blindly trust results for these large values of $N$, and we believe that CONAN should be run for no more than approximately 35 particles. 

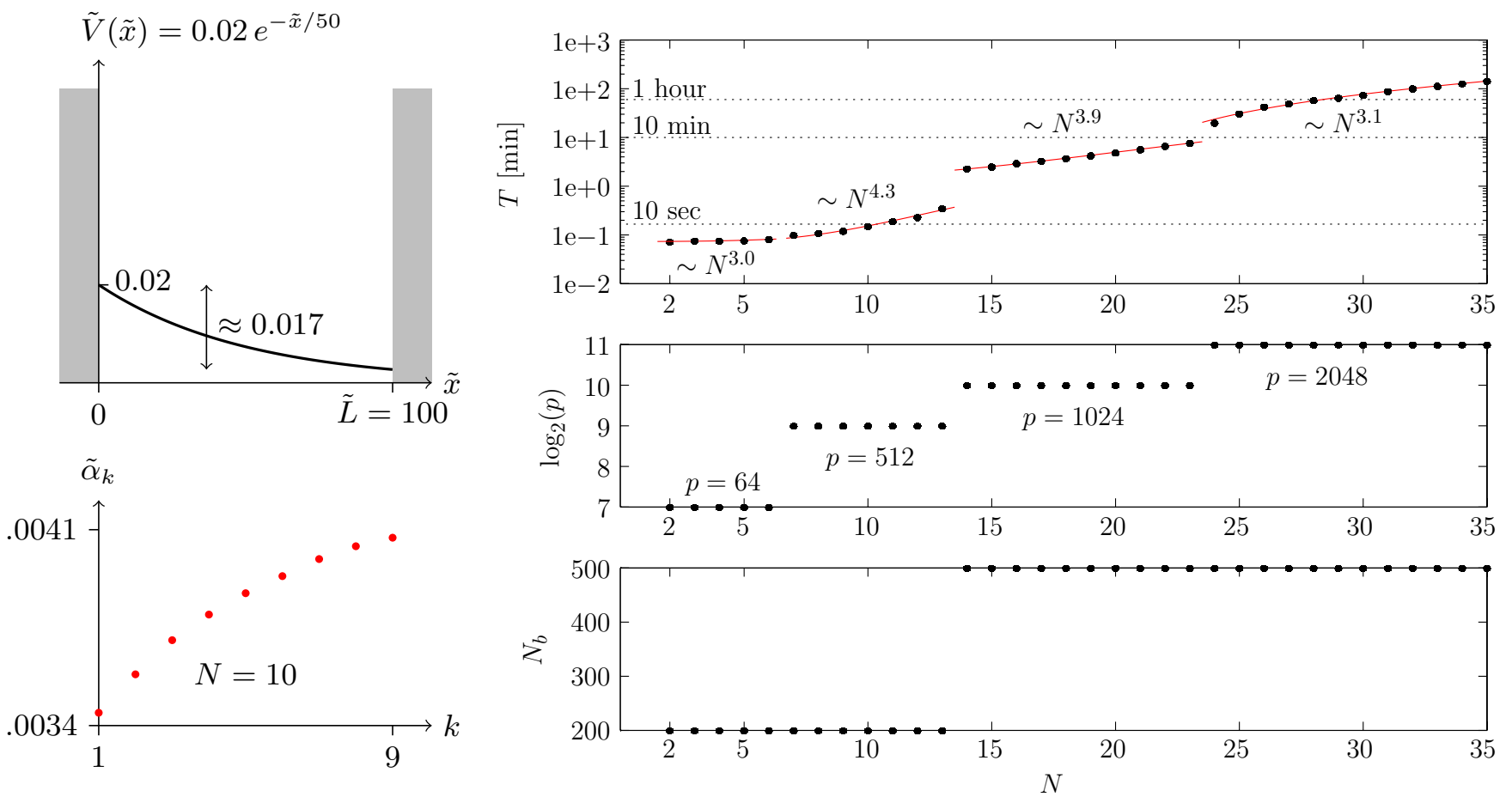

FIG. 3: Asymmetric tilted potential in a box. To the left we sketch the potential submitted to CONAN and the calculated geometric coefficients for $N=10$. To the right, on the top panel, we plot the computation time $T$ versus the number of particles $N$ including the scaling for a fixed $p$. Below we show the chosen bit precision $p$ (middle) and basis size $N_{b}$ (bottom). The estimated error is $0.0001 \%$ or less for $N \lesssim 28$, but increases to at most $3 \%$ for $N=35$.

\section{B. Asymmetric tilted potential in a box}

Here we consider a simple box potential with an added exponentially decaying potential. Therefore, the resulting potential is not symmetric around the center of the box, but tilted to one side, i.e.,

$$
V(x)= \begin{cases}0.02 \varepsilon e^{-\frac{x}{50 \ell}} & \text { if } 0<x<L=100 \ell \\ \infty & \text { otherwise }\end{cases}
$$

where the numerical factors have been chosen such that the difference $V(0)-V(L) \approx 0.017 \varepsilon$ is comparable with the energy scale of the system, and the energy unit $\varepsilon$ used by CONAN is given by Eq. (16).

We submit to CONAN the following dimensionless potential

$$
\tilde{V}(\tilde{x})=0.02 e^{-\tilde{x} / 50} .
$$

We calculate the geometric coefficients for $N=2, \ldots, 35$ with the basis size $N_{b}$ and bit precision $p$ chosen to retain a high degree of precision on the results. The corresponding computation time is shown in Fig. 3 (top) for $p=64,512$, 1024 and 2048.

We found that $N_{b}=200$ was sufficient to ensure an error below $0.0001 \%$ for $N<14$, for more particles the basis size had to been increased to retain a high degree of precision. By comparing $\alpha_{k}$ with $\alpha_{N-k}$ calculated for the mirror reflected potential $V(L-x)$, we estimate an error of $0.0001 \%$ for $N=28$ for the most inaccurate coefficients. This increases to $0.001 \%$ for $N=30$ and $3 \%$ for $N=35$.

\section{CONCLUSIONS}

We presented an algorithm for computing the local exchange coefficients for a strongly interacting $N$-particle system confined to one dimension by an external potential. We discussed the numerical implementation of the method in a piece of open source software, CONAN. Then, we discussed the use of the program, including examples and estimates of the precision of the results. We found that CONAN could produce reliable results for up to around $N=35$, and that the computation time typically scales as $O\left(N^{3.5 \pm 0.4}\right)$. Computation times were around 10 seconds for $N=10$ and less than 10 minutes for $N=20$. The approach described here may be extended in a straightforward manner to compute, for instance, densities in strongly interacting systems or correlation functions.

Remark: While preparing this paper we became aware of a recent paper by Deuretzbacher et al. [52] which presents a method for computing the coefficients that has some similarities to the one used here. We note that while Deuretzbacher et al. use a fitting method with Chebyshev polynomials to get high-order derivatives, we 
use a recursive formula as described in the appendix. This could have influence on the numerical stability of either algorithm. It would be very interesting to explore whether one may combine these two different approach to achieve even larger stability than currently available in either approach.

\section{Acknowledgments}

We would like to thank M. Valiente, A. S. Jensen, D. V. Fedorov, O. V. Marchukov, and D. Petrosyan for collaboration on the spin model approach. We thank E. M. Eriksen and E. J. Lindgren for discussions on early developments on a formula to compute the local exchange coefficients, as well as J. M. Midtgaard and A. A. S. Kalaee for help in testing these developments in spin systems. A. G. V. and N. T. Z. acknowledge support from the Danish Council for Independent Research DFF Natural Sciences Sapere Aude program, and A. G. V. acknowledges partial support from the Helmholtz Association under contract HA216/EMMI. N. J. S. L., L. B. K., A. E. T., and N. T. Z. acknowledge support by grants from the Carlsberg Foundation. The development of CONAN was assisted by J. Termansen and J. H. Jensen with support from the Carlsberg Foundation.

\section{Appendix A: Derivation of Eq. (6)}

In this appendix we derive Eq. (6) from Eq. (5). We start by writing the Slater determinant that defines the wave function for the system of $N$ spinless fermions using the Leibniz formula for determinants

$$
\Phi_{0}\left(x_{1}, \ldots, x_{N}\right)=\sum_{\pi} \operatorname{sign}(\pi) \prod_{i=1}^{N} \psi_{\pi(i)}\left(x_{i}\right),
$$

here $\pi$ denotes the permutation operator that acts on the set of first $N$ natural numbers $\{1,2, \ldots, N\}$, and $\psi_{j}$ is the $j$ th normalised one-body wave function defined in the main text. Next, we write $\alpha_{k}$ as

$$
\alpha_{k}=\sum_{i=1}^{N} \sum_{j=1}^{N}(-1)^{i+j} \int_{a}^{b} \mathrm{~d} x_{k} \frac{\partial \psi_{i}\left(x_{k}\right)}{\partial x_{k}} \frac{\partial \psi_{j}\left(x_{k}\right)}{\partial x_{k}} \int_{a \leq x_{1}<x_{2}<\cdots<x_{N-1} \leq b} \mathrm{~d} x_{1} \ldots \mathrm{d} x_{k-1} \mathrm{~d} x_{k+1} \ldots \mathrm{d} x_{N-1}\left(\xi^{i} \xi^{j}\right)\left(x_{1}, \ldots, x_{N-1}\right),
$$

where the interval $[a, b]$ is the support of the trapping potential, see the main text for details. To obtain this expression we used the Laplace expression for $\Phi_{0}$,

$$
\Phi_{0}=\sum_{i=1}^{N}(-1)^{i+N} \psi_{i}\left(x_{N}\right) \xi^{i}\left(x_{1}, \ldots, x_{N-1}\right),
$$

where $\xi^{i}\left(x_{1}, \ldots, x_{N-1}\right)=\sum_{\pi_{i}} \operatorname{sign}\left(\pi_{i}\right) \prod_{j=1}^{N-1} \psi_{\pi_{i}(j)}\left(x_{j}\right), \pi_{i}$ is the permutation operator defined on the set of $N-1$ elements: $\{1, \ldots, N\} \backslash i$. To produce Eq. (6) we simplify the inner integral

$$
I_{k, i j}=\int_{x_{1}<x_{2}<\cdots<x_{N-1}} \mathrm{~d} x_{1} \ldots \mathrm{d} x_{k-1} \mathrm{~d} x_{k+1} \ldots \mathrm{d} x_{N-1}\left(\xi^{i} \xi^{j}\right)\left(x_{1}, \ldots, x_{N-1}\right) .
$$

For this we note that $\xi^{i} \xi^{j}$ is a symmetric function, i.e., $\left(\xi^{i} \xi^{j}\right)\left(\ldots, x_{k}, \ldots, x_{l}, \ldots\right)=\left(\xi^{i} \xi^{j}\right)\left(\ldots, x_{l}, \ldots, x_{k}, \ldots\right)$, which allows us to change the integration limits as

$$
I_{k, i j}=\frac{1}{(k-1) !(N-1-k) !} \int_{a}^{x_{k}} \mathrm{~d} x_{1} \int_{a}^{x_{k}} \mathrm{~d} x_{2} \ldots \int_{a}^{x_{k}} \mathrm{~d} x_{k-1} \int_{x_{k}}^{b} \mathrm{~d} x_{k+1} \ldots \int_{x_{k}}^{b} \mathrm{~d} x_{N-1} \xi^{i} \xi^{j} .
$$

Throughout our investigation we noticed that similar integrals appear also for observables. Therefore, we find it useful to consider a more general integral, that has a similar structure

$$
P_{l}^{n}(c)[F \Phi]=\int_{a}^{c} \mathrm{~d} x_{1} \ldots \int_{a}^{c} \mathrm{~d} x_{l-1} \int_{c}^{b} \mathrm{~d} x_{l} \ldots \int_{c}^{b} \mathrm{~d} x_{n} F\left(x_{1}, \ldots, x_{n}\right) \Phi\left(x_{1}, \ldots, x_{n}\right),
$$

where $l-1$ integrals should be taken from $a$ to $c$, other integrals are taken from $c$ to $b$, the superscript $n$ defines the number of variables. Functions $F$ and $\Phi$ are determinants build with the orthonormalized sets of functions $\left\{f_{i}\right\}$ and $\left\{\phi_{i}\right\}$ correspondingly. That is 


$$
\Phi\left(x_{1}, x_{2}, \ldots, x_{n}\right)=\left|\begin{array}{cccc}
\phi_{1}\left(x_{1}\right) & \phi_{2}\left(x_{1}\right) & \cdots & \phi_{n}\left(x_{1}\right) \\
\phi_{1}\left(x_{2}\right) & \phi_{2}\left(x_{2}\right) & \cdots & \phi_{n}\left(x_{2}\right) \\
\vdots & \vdots & \ddots & \vdots \\
\phi_{1}\left(x_{n}\right) & \phi_{2}\left(x_{n}\right) & \cdots & \phi_{n}\left(x_{n}\right)
\end{array}\right| \text {, and } F\left(x_{1}, x_{2}, \ldots, x_{n}\right)=\left|\begin{array}{cccc}
f_{1}\left(x_{1}\right) & f_{2}\left(x_{1}\right) & \cdots & f_{n}\left(x_{1}\right) \\
f_{1}\left(x_{2}\right) & f_{2}\left(x_{2}\right) & \cdots & f_{n}\left(x_{2}\right) \\
\vdots & \vdots & \ddots & \vdots \\
f_{1}\left(x_{n}\right) & f_{2}\left(x_{n}\right) & \cdots & f_{n}\left(x_{n}\right)
\end{array}\right| \text {. }
$$

Let us first consider $P_{n+1}^{n}(c)[F \Phi]$. Noticing that both $F$ and $\Phi$ are fully antisymmetric functions in their variables, we write $P_{n+1}^{n}$ as

$$
\begin{aligned}
P_{n+1}^{n}(c)[F \Phi] & =n ! \int_{a}^{c} \mathrm{~d} x_{1} \ldots \int_{a}^{c} \mathrm{~d} x_{n} f_{1}\left(x_{1}\right) f_{2}\left(x_{2}\right) \ldots f_{n}\left(x_{n}\right)\left|\begin{array}{cccc}
\phi_{1}\left(x_{1}\right) & \phi_{2}\left(x_{1}\right) & \cdots & \phi_{n}\left(x_{1}\right) \\
\phi_{1}\left(x_{2}\right) & \phi_{2}\left(x_{2}\right) & \cdots & \phi_{n}\left(x_{2}\right) \\
\vdots & \vdots & \ddots & \vdots \\
\phi_{1}\left(x_{n}\right) & \phi_{2}\left(x_{n}\right) & \cdots & \phi_{n}\left(x_{n}\right)
\end{array}\right| \\
& =n ! \int_{a}^{c} \mathrm{~d} x_{1} \ldots \int_{a}^{c} \mathrm{~d} x_{n}\left|\begin{array}{cccc}
f_{1}\left(x_{1}\right) \phi_{1}\left(x_{1}\right) & f_{1}\left(x_{1}\right) \phi_{2}\left(x_{1}\right) & \cdots & f_{1}\left(x_{1}\right) \phi_{n}\left(x_{1}\right) \\
f_{2}\left(x_{2}\right) \phi_{1}\left(x_{2}\right) & f_{2}\left(x_{2}\right) \phi_{2}\left(x_{2}\right) & \cdots & f_{2}\left(x_{2}\right) \phi_{n}\left(x_{2}\right) \\
\vdots & \vdots & \ddots & \vdots \\
f_{n}\left(x_{n}\right) \phi_{1}\left(x_{n}\right) & f_{n}\left(x_{n}\right) \phi_{2}\left(x_{n}\right) & \cdots & f_{n}\left(x_{n}\right) \phi_{n}\left(x_{n}\right)
\end{array}\right|,
\end{aligned}
$$

the integration can be performed inside of the determinant, which leads to $P_{n+1}^{n}(c)[F \Phi]=n ! \operatorname{det} A(c)$ where the matrix elements in $A(c)$ are defined as $[A(c)]_{i, j}=\int_{a}^{c} \mathrm{~d} x f_{i}(x) \phi_{j}(x)$.

Next we consider $P_{n}^{n}$

$$
\begin{aligned}
P_{n}^{n}(c)[F \Phi] & =\int_{a}^{c} \mathrm{~d} x_{1} \ldots \int_{a}^{c} \mathrm{~d} x_{n-1} \int_{c}^{b} \mathrm{~d} x_{n} F\left(x_{1}, \ldots, x_{n}\right) \Phi\left(x_{1}, \ldots, x_{n}\right) \\
& =\sum_{i=1}^{n} \sum_{j=1}^{n}(-1)^{i+j} \int_{c}^{b} \mathrm{~d} x_{n} f_{i}\left(x_{n}\right) \phi_{j}\left(x_{n}\right) P_{n}^{n-1}(c)\left[F^{i} \Phi^{j}\right] \\
& =(n-1) ! \sum_{i=1}^{n} \sum_{j=1}^{n}(-1)^{i+j} \int_{c}^{b} \mathrm{~d} x_{n} f_{i}\left(x_{n}\right) \phi_{j}\left(x_{n}\right) \operatorname{det}\left[(A(c))^{(i j)}\right],
\end{aligned}
$$

where the functions $F^{i}$ and $\Phi^{j}$ are obtained from the Laplace expansions of $F$ and $\Phi$ respectively, the matrix $(A(c))^{(i j)}$ is obtained from the matrix $A(c)$ by crossing out the $i^{\prime}$ th row and $j^{\prime}$ th column (i.e., $A^{(i j)}$ is the $i j^{\prime}$ 'th submatrix). From here we can proceed by two different paths: First we can notice that the expression in Eq. A8) can be rewritten using Jacobi's formula for the derivative of the determinant $\operatorname{as} \operatorname{tr}(T \operatorname{adj}(A))=\left.\frac{\partial \operatorname{det}(A+\lambda T)}{\partial \lambda}\right|_{\lambda=0}$, where the matrix $T$ is defined as $[T]_{i, j}=\int_{c}^{b} \mathrm{~d} x f_{i}(x) \phi_{j}(x)$, and $\operatorname{adj} A$ denotes the adjugate of $A$. This observation allows us to derive the identity $P_{n}^{n}(c)[F \Phi]=\left.(n-1) ! \frac{\partial \operatorname{det}(A+\lambda T)}{\partial \lambda}\right|_{\lambda=0}$. By repeating the same steps (i.e., first is to write $P_{j}^{n}$ using $P_{j}^{n-1}$, second is to use Jacobi's formula) for $P_{n-1}^{n}, P_{n-2}^{n}, \ldots$ we obtain $P_{l}^{n}(c)[F \Phi]=\left.(l-1) ! \frac{\partial^{n-l+1} \operatorname{det}(A+\lambda T)}{\partial \lambda^{n-l+1}}\right|_{\lambda=0}$.

Another path, which was used to derive Eq. (6), rests on the assumption that $f_{i}$ and $\phi_{j}$ are orthonogonal to each other, i.e., $\int_{a}^{b} \mathrm{~d} x f_{i}(x) \phi_{j}(x)=\delta_{i j}$, where $\delta_{i j}$ is Kronecker's delta. This assumption allows us to rewrite Eq. A8) as

$$
\begin{aligned}
P_{n}^{n}(c)[F \Phi] & =(n-1) ! \sum_{i=1}^{n} \sum_{j=1}^{n}(-1)^{i+j} \operatorname{det} A^{(i j)}\left(\delta_{i j}-[A]_{i, j}\right) \\
& =-(n-1) !\left(\left.\frac{\partial \operatorname{det}(A-\lambda \mathbf{I})}{\partial \lambda}\right|_{\lambda=0}+n \operatorname{det} A\right) .
\end{aligned}
$$

Let us now consider $P_{n-1}^{n}(c)$

$$
\begin{aligned}
P_{n-1}^{n}(c)[F \Phi] & =\sum_{i=1}^{n} \sum_{j=1}^{n}(-1)^{i+j} \int_{c}^{b} \mathrm{~d} x_{n} f_{i}\left(x_{n}\right) \phi_{j}\left(x_{n}\right) P_{n-1}^{n-1}(c)\left[F^{i} \Phi^{j}\right] \\
& =\sum_{i=1}^{n} \sum_{j=1}^{n} P_{n-1}^{n-1}(c)\left[F^{i} \Phi^{j}\right] \delta_{i j}-P_{n}^{n}(c)[F \Phi] \\
& =(n-2) !\left[\left.\frac{\partial^{2} \operatorname{det}(A-\lambda \mathbf{I})}{\partial \lambda^{2}}\right|_{\lambda=0}+\left.(n-1) \frac{\partial \operatorname{det}(A-\lambda \mathbf{I})}{\partial \lambda}\right|_{\lambda=0}\right]+(n-1) !\left[\left.\frac{\partial \operatorname{det}(A-\lambda \mathbf{I})}{\partial \lambda}\right|_{\lambda=0}+n \operatorname{det} A\right] .
\end{aligned}
$$


The pattern for $P_{l}^{n}(c)$ can be guessed now, and we write $P_{l}^{n}$ as

$$
P_{l}^{n}(c)[F \Phi]=\left.(-1)^{n+1-l} \sum_{i=0}^{n+1-l}(n-i) !\left(\begin{array}{c}
n+1-l \\
i
\end{array}\right) \frac{\partial^{i} \operatorname{det}(A(c)-\lambda \mathbf{I})}{\partial \lambda^{i}}\right|_{\lambda=0} .
$$

One can easily check, e.g., by induction, that this expression is correct. The meaning of the factors in the sum can be understood from the expression for $P_{n-1}^{n}$; the $(n-i)$ ! factor always comes with the $i$ th derivative, whereas the $\left(\begin{array}{c}n+1-l \\ i\end{array}\right)$ factor shows how many terms with the $i$ 'th derivative are in the expression.

Now, we use the expression for $P_{l}$ to obtain $\alpha_{k}$. Firstly, we write $I_{k, i j}$ as

$$
I_{k, i j}=\frac{1}{(k-1) !(N-1-k) !} \int_{a}^{x_{k}} \mathrm{~d} x_{1} \int_{a}^{x_{k}} \mathrm{~d} x_{2} \ldots \int_{a}^{x_{k}} \mathrm{~d} x_{k-1} \int_{x_{k}}^{b} \mathrm{~d} x_{k+1} \ldots \int_{x_{k}}^{b} \mathrm{~d} x_{N-1} F\left(x_{1}, \ldots, x_{N-1}\right) \Phi\left(x_{1}, \ldots, x_{N-1}\right)
$$

where the functions $F$ and $\Phi$ are defined above, assuming that $n=N-1$, and $\left\{f_{1}, \ldots, f_{N-1}\right\}=$ $\left\{\psi_{1}, \ldots, \psi_{i-1}, \psi_{i+1}, \ldots, \psi_{N}\right\}$ and $\left\{\phi_{1}, \ldots, \phi_{N-1}\right\}=\left\{\psi_{1}, \ldots, \psi_{j-1}, \psi_{j+1}, \ldots, \psi_{N}\right\}$. Secondly, we rewrite $I_{k, i j}$ as

$$
I_{k, i j}=\sum_{l, m}(-1)^{l+m} \frac{f_{l}\left(x_{k}\right) \phi_{m}\left(x_{k}\right)}{(k-1) !(N-1-k) !} \int_{a}^{x_{k}} \mathrm{~d} x_{1} \int_{a}^{x_{k}} \mathrm{~d} x_{2} \ldots \int_{a}^{x_{k}} \mathrm{~d} x_{k-1} \int_{x_{k}}^{b} \mathrm{~d} x_{k+1} \ldots \int_{x_{k}}^{b} \mathrm{~d} x_{N-1} F^{l} \Phi^{m}
$$

where $\Phi^{m}\left(F^{m}\right)$ is obtained from $\Phi(F)$ by crossing out the $k$ th row and $m$ th column. The integral can be easily taken, using the results from Eq. A11, i.e.,

$$
I_{k, i j}=\left.\sum_{l, m} \frac{f_{l}\left(x_{k}\right) \phi_{m}\left(x_{k}\right)}{(k-1) !(N-1-k) !}(-1)^{N-k-1+l+m} \sum_{r=0}^{N-k-1}(N-2-r) !\left(\begin{array}{c}
N-k-1 \\
r
\end{array}\right) \frac{\partial^{r} \operatorname{det}\left(\left(A\left(x_{k}\right)\right)^{(l m)}-\lambda \mathbf{I}^{(i j)}\right)}{\partial \lambda^{r}}\right|_{\lambda=0},
$$

where we use $\mathbf{I}^{(i j)}$, because in this case $\int_{a}^{b} \mathrm{~d} x f_{l}(x) \phi_{m}(x)=\left[\mathbf{I}^{(i j)}\right]_{l, m}$. Now we note that $\phi_{m}(x) f_{l}(x)=\frac{\partial[A(x)]_{l, m}}{\partial x}$ and use Jacobi's formula to obtain the expression for $\alpha_{k}$

$$
\alpha_{k}=\sum_{i=1}^{N} \sum_{j=1}^{N} \sum_{l=0}^{N-1-k} \frac{(-1)^{i+j+N-1-k}}{l !}\left(\begin{array}{c}
N-l-2 \\
k-1
\end{array}\right) \int_{a}^{b} \mathrm{~d} x \frac{\mathrm{d} \psi_{i}}{\mathrm{~d} x} \frac{\mathrm{d} \psi_{j}}{\mathrm{~d} x} \frac{\mathrm{d}}{\mathrm{d} x}\left[\frac{\partial^{l}}{\partial \lambda^{l}} \operatorname{det}\left[(B(x)-\lambda \mathbf{I})^{(i j)}\right]\right]_{\lambda=0},
$$

where $B(x)$ is the symmetric matrix defined in Eq. (7). Next we integrate by parts to eliminate the derivative of the expression in square brackets. This procedure yields

$\alpha_{k}=2 \sum_{i=1}^{N} \sum_{j=1}^{N} \sum_{l=0}^{N-1-k} \frac{(-1)^{i+j+N-k}}{l !}\left(\begin{array}{c}N-l-2 \\ k-1\end{array}\right) \int_{a}^{b} \mathrm{~d} x \frac{2 m}{\hbar^{2}}\left(V(x)-E_{i}\right) \psi_{i}(x) \frac{\mathrm{d} \psi_{j}}{\mathrm{~d} x}\left[\frac{\partial^{l}}{\partial \lambda^{l}} \operatorname{det}\left[(B(x)-\lambda \mathbf{I})^{(i j)}\right]\right]_{\lambda=0}+\mathcal{B}$,

where $\mathcal{B}$ denotes the boundary term arising from the partial integration

$$
\mathcal{B}=\left.\sum_{i=1}^{N} \sum_{j=1}^{N} \sum_{l=0}^{N-1-k} \frac{(-1)^{i+j+N-1-k}}{l !}\left(\begin{array}{c}
N-l-2 \\
k-1
\end{array}\right) \frac{\mathrm{d} \psi_{i}}{\mathrm{~d} x} \frac{\mathrm{d} \psi_{j}}{\mathrm{~d} x}\left[\frac{\partial^{l}}{\partial \lambda^{l}} \operatorname{det}\left[(B(x)-\lambda \mathbf{I})^{(i j)}\right]\right]_{\lambda=0}\right|_{a} ^{b} .
$$

Note that to obtain this equation we use the Schrödinger equation from Eq. (3). The boundary term in Eq. (A17) can be simplified significantly. To do this we consider

$$
K_{i j}(x)=\left[\frac{\partial^{l}}{\partial \lambda^{l}} \operatorname{det}\left[(B(x)-\lambda \mathbf{I})^{(i j)}\right]\right]_{\lambda=0},
$$

evaluated at the boundary points $x=a$ and $x=b$, where the matrix $B(x)$ reduces to $B(a)=\mathbf{0}$ or $B(b)=\mathbf{I}$. Therefore, $K_{i j}(x)$ at $x=a$ and $x=b$ can be written as

$$
K_{i j}(a)=\left[\frac{\partial^{l}}{\partial \lambda^{l}} \operatorname{det}\left[(-\lambda \mathbf{I})^{(i j)}\right]\right]_{\lambda=0}, \quad K_{i j}(b)=\left[\frac{\partial^{l}}{\partial \lambda^{l}} \operatorname{det}\left[((1-\lambda) \mathbf{I})^{(i j)}\right]\right]_{\lambda=0} .
$$


If $i=j$ then $\mathbf{I}^{(i j)}$ is the $(N-1) \times(N-1)$ identity matrix, however if $i \neq j$ the matrix has a zero-row (and zero-column), so its determinant is 0 . Thus, $\operatorname{det} \mathbf{I}^{(i j)}$ is a Kronecker delta, $\delta_{i j}$. This observation leads to the following expressions

$$
K_{i j}(a)=\left[\frac{\partial^{l}}{\partial \lambda^{l}}(-\lambda)^{N-1} \delta_{i j}\right]_{\lambda=0}, K_{i j}(b)=\left[\frac{\partial^{l}}{\partial \lambda^{l}}(1-\lambda)^{N-1} \delta_{i j}\right]_{\lambda=0} .
$$

Now let us evaluate these expressions. At the lower limit we have

$$
K_{i j}(a)=\left.(-1)^{l} \frac{(N-1) !}{(N-l-1) !}(-\lambda)^{N-l-1} \delta_{i j}\right|_{\lambda=0}=(-1)^{l} \frac{(N-1) !}{(N-l-1) !} \delta_{N-l-1,0} \delta_{i j}
$$

Notice that $N-l-1 \geq N-(N-1-k)-1 \geq N-(N-2)-1=1>0$ for all terms in the sum over $l$ in Eq. A17), so $K_{i j}(a)=0$ there. At the upper limit we have

$$
K_{i j}(b)=\left.(-1)^{l} \frac{(N-1) !}{(N-l-1) !}(1-\lambda)^{N-l-1} \delta_{i j}\right|_{\lambda=0}=(-1)^{l} \frac{(N-1) !}{(N-l-1) !} \delta_{i j} .
$$

Inserting this result into Eq. A17, we obtain the boundary term in the form

$$
\mathcal{B}=\sum_{i=1}^{N}\left[\frac{\mathrm{d} \psi_{i}}{\mathrm{~d} x}\right]_{x=b}^{2} \sum_{l=0}^{N-1-k}(-1)^{N-1-k+l}\left(\begin{array}{c}
N-l-2 \\
k-1
\end{array}\right) \frac{(N-1) !}{(N-l-1) ! l !},
$$

which simplifies as the sum over $l$ equals unity. This can be proven using the binomial theorem and the definition of the beta function.

$$
\begin{aligned}
& \sum_{l=0}^{N-1-k}(-1)^{N-1-k+l}\left(\begin{array}{c}
N-l-2 \\
k-1
\end{array}\right) \frac{(N-1) !}{(N-l-1) ! l !} \\
& =\frac{(N-1) !}{(N-1-k) !(k-1) !} \sum_{l=0}^{N-1-k}(-1)^{N-1-k+l}\left(\begin{array}{c}
N-1-k \\
l
\end{array}\right) \frac{1}{N-l-1} \\
& =\frac{(N-1) !}{(N-1-k) !(k-1) !} \sum_{l=0}^{N-1-k}(-1)^{N-1-k+l}\left(\begin{array}{c}
N-1-k \\
l
\end{array}\right) \int_{0}^{1} \mathrm{~d} x x^{N-l-2} \\
& =\frac{(N-1) !}{(N-1-k) !(k-1) !} \int_{0}^{1} \mathrm{~d} x x^{k-1} \sum_{l=0}^{N-1-k}\left(\begin{array}{c}
N-1-k \\
l
\end{array}\right)(-x)^{N-1-k-l} \\
& =\frac{(N-1) !}{(N-1-k) !(k-1) !} \int_{0}^{1} \mathrm{~d} x x^{k-1}(1-x)^{N-k-1} \\
& =1 \text {, }
\end{aligned}
$$

where we have recognized the last integral as the beta function. Thus, we finally have derived Eq. 6.

\section{Appendix B: Derivation of Eq. (11)}

Here we prove the equality

$$
\left.\frac{\partial^{l}}{\partial \lambda^{l}} \operatorname{det}\left[(B(x)-\lambda \mathbf{I})^{(i j)}\right]\right|_{\lambda=0}=(-1)^{i+j} l ! \mathbf{u}_{j}^{\mathrm{T}}\left(\sum_{n=0}^{l} p_{l-n} D^{-(n+1)}\right) \mathbf{u}_{i}
$$

presented in Sec. III.

By choosing real valued wave functions one can ensure that $B$ is a real and symmetric matrix. By the spectral theorem it is then possible to diagonalize it using an orthogonal matrix $U=\left(\mathbf{u}_{1} \ldots \mathbf{u}_{N}\right)$ such that $B=U^{\mathrm{T}} D U$ with $D$ being a diagonal matrix containing the eigenvalues of $B$. Let us denote by $U_{n}=\left(\mathbf{u}_{1} \ldots \mathbf{u}_{n-1} \mathbf{u}_{n+1} \ldots \mathbf{u}_{N}\right)$, i.e., $U_{n}$ is the matrix $U$ with the $n$ 'th column removed. In this notation the $i j$ 'th submatrix in Eq. B1 is simply

$$
(B-\lambda \mathbf{I})^{(i j)}=\left(U^{\mathrm{T}}(D-\lambda \mathbf{I}) U\right)^{(i j)}=U_{i}^{\mathrm{T}}(D-\lambda \mathbf{I}) U_{j} .
$$


To proceed further we note that it is possible to turn $U_{i}^{\mathrm{T}}$ and $U_{j}$ into square matrices (by inserting respectively an extra row and column) without changing the value of the determinant. This is possible when $D-\lambda \mathbf{I}$ is invertible in a small region around $\lambda=0$ which is the case as long as all eigenvalues of $B$ are non-zero. In this case

$$
\left(\begin{array}{c}
\mathbf{u}_{i}^{\mathrm{T}} \\
U_{i}^{\mathrm{T}}
\end{array}\right)(D-\lambda \mathbf{I})\left(\left[(D-\lambda \mathbf{I})^{-1} \mathbf{u}_{i}\right] \quad U_{j}\right)=\left(\begin{array}{cc}
1 & \mathbf{u}_{i}^{\mathrm{T}}(D-\lambda \mathbf{I}) U_{j} \\
\mathbf{0} & (B-\lambda \mathbf{I})^{(i j)}
\end{array}\right),
$$

where we used that $U$ is an orthogonal matrix. One can prove that the determinant is indeed unchanged by expanding the determinant in the first column of the RHS of the above expression. As the product matrices are now square the determinant can be evaluated using the product rule for determinants, hence

$$
\operatorname{det}\left[(B-\lambda \mathbf{I})^{(i j)}\right]=\operatorname{det}\left(\begin{array}{c}
\mathbf{u}_{i}^{\mathrm{T}} \\
U_{i}^{\mathrm{T}}
\end{array}\right) \operatorname{det}(D-\lambda \mathbf{I}) \operatorname{det}\left((D-\lambda \mathbf{I})^{-1} \mathbf{u}_{i} U_{j}\right),
$$

we rearrange the first matrix on the RHS utilizing the fact that a swap of rows changes the sign of determinant

$$
\begin{aligned}
\operatorname{det}\left[(B-\lambda \mathbf{I})^{(i j)}\right] & =(-1)^{i+j} \operatorname{det}(D-\lambda \mathbf{I}) \operatorname{det}\left(\begin{array}{c}
\mathbf{u}_{j}^{\mathrm{T}} \\
U_{j}^{\mathrm{T}}
\end{array}\right) \operatorname{det}\left((D-\lambda \mathbf{I})^{-1} \mathbf{u}_{i} U_{j}\right) \\
& =(-1)^{i+j} \operatorname{det}(D-\lambda \mathbf{I}) \operatorname{det}\left(\begin{array}{cc}
\mathbf{u}_{j}^{\mathrm{T}}(D-\lambda \mathbf{I})^{-1} \mathbf{u}_{i} & 0 \\
U_{j}^{\mathrm{T}}(D-\lambda \mathbf{I})^{-1} \mathbf{u}_{i} & \mathbf{I}
\end{array}\right) \\
& =(-1)^{i+j} \operatorname{det}(D-\lambda \mathbf{I})\left[\mathbf{u}_{j}^{\mathrm{T}}(D-\lambda \mathbf{I})^{-1} \mathbf{u}_{i}\right] .
\end{aligned}
$$

We find this expression more agreeable than the expression we began with, as $D-\lambda \mathbf{I}$ is a diagonal matrix.

From here on the proof of Eq. (B1) is straightforward. First we note that

$$
\left.\frac{\mathrm{d}^{n}}{\mathrm{~d} \lambda^{n}}(D-\lambda \mathbf{I})^{-1}\right|_{\lambda=0}=n ! D^{-(n+1)}
$$

furthermore, the determinant is simply a polynomial in $\lambda$

$$
\operatorname{det}(D-\lambda \mathbf{I})=p_{N} \lambda^{N}+\ldots+p_{1} \lambda+p_{0},
$$

from which we find that

$$
\left.\frac{\mathrm{d}^{n}}{\mathrm{~d} \lambda^{n}} \operatorname{det}(D-\lambda \mathbf{I})\right|_{\lambda=0}=n ! p_{n}
$$

The coefficients $p_{k}$ are found from the product $\prod_{i}\left(D_{i i}-1\right)$. To optimize the calculation we introduce $p_{l}^{(r)}$, which is the coefficient obtained through the multiplication of the first $r$ factors in $\prod_{i}\left(D_{i i}-1\right)$. Thus, e.g., $p_{0}^{(1)}=D_{11}$ and $p_{1}^{(1)}=-1$. The computation of $p_{k}$ is then performed iteratively

$$
p_{l+1}^{(r+1)}=d_{r+1} p_{l+1}^{(r)}-p_{l}^{(r)}
$$

This procedure at $r=N$ gives the coefficients of the polynomial arising from the determinant. The computation is done in $O\left(N^{2}\right)$ steps. With this all parts of the final expression for the derivatives of the determinants are ready. As advertised it is

$$
\begin{aligned}
{\left[\frac{\partial^{l}}{\partial \lambda^{l}} \operatorname{det}\left[(B(x)-\lambda \mathbf{I})^{(i j)}\right]\right]_{\lambda=0} } & =(-1)^{i+j} \sum_{n=0}^{l}\left(\begin{array}{l}
l \\
n
\end{array}\right)(l-n) ! p_{l-n} n ! \mathbf{u}_{j}^{\mathrm{T}} D^{-(n+1)} \mathbf{u}_{i} \\
& =(-1)^{i+j} l ! \mathbf{u}_{j}^{\mathrm{T}}\left(\sum_{n=0}^{l} p_{l-n} D^{-(n+1)}\right) \mathbf{u}_{i} .
\end{aligned}
$$

Finally, let us justify the assumption that none of the eigenvalues of $B(x)$ is zero, because the entire procedure hinges on this fact. Assume for contradiction that there exists a non-zero vector $\mathbf{v}$ so that $B \mathbf{v}=\mathbf{0}$, i.e.,

$$
\sum_{j=1}^{N}[B(x)]_{i j} v_{j}=\int_{a}^{x} \mathrm{~d} y \psi_{i}(y) \sum_{j=1}^{N} v_{j} \psi_{j}(y)=0, \quad \forall i \leq N
$$


This holds for all $i$, therefore, it must hold for linear combinations too. Hence,

$$
0=\sum_{i=1}^{N} v_{i} \int_{a}^{x} \mathrm{~d} y \psi_{i}(y) \sum_{j=1}^{N} v_{j} \psi_{j}(y)=\int_{a}^{x} \mathrm{~d} y\left(\sum_{i=1}^{N} v_{i} \psi_{i}(y)\right)^{2} \Rightarrow \sum_{i=1}^{N} v_{i} \psi_{i}(y)=0, \quad y \in[a, x] .
$$

As $\mathbf{v}$ is non-zero, it should contain at least one non-zero entry. Without loss of generality, let us assume $v_{1} \neq 0$ (we can always redefine the index $i$ such that $v_{1} \neq 0$ ). The wave functions individually solve the one-body Schrödinger equation, therefore

$$
\begin{aligned}
\prod_{k=2}^{N}\left(H_{0}-E_{k}\right) \sum_{i=1}^{N} v_{i} \psi_{i}(y) & =\sum_{i=1}^{N}\left(E_{i}-E_{2}\right)\left(E_{i}-E_{3}\right) \cdots\left(E_{i}-E_{N}\right) v_{i} \psi_{i}(y) \\
& =\left(E_{1}-E_{2}\right)\left(E_{1}-E_{3}\right) \cdots\left(E_{1}-E_{N}\right) v_{1} \psi_{1}(y)=0, \quad y \in[a, x]
\end{aligned}
$$

It is known that a one-body system in a one-dimensional trap without singularities has a non-degenerate spectrum, i.e., all the $E_{i}$ 's are distinct, therefore the factor $\left(E_{1}-E_{2}\right) \cdots\left(E_{1}-E_{N}\right)$ does not vanish. By assumption $v_{1} \neq 0$, thus, $\psi_{1}(y)$ must vanish on $[a, x]$. Now consider the differential equation

$$
\left(H_{0}-E_{1}\right) \psi_{1}(y)=0, \quad y \in[a, b]
$$

which is fulfilled because $\left(H_{0}-E_{1}\right)$ acts as the zero operator on the entire interval $[a, b]$. The same differential equation is solved by the zero function on $[a, b]$. Now as the solutions $\psi_{1}(y)$ and 0 (the zero function) coincide on the subinterval $[a, x]$ it follows from the theory of ordinary differential equations that given sufficiently smooth $V$, the two functions must be identical on the whole interval $[a, b]$. We are forced to conclude that

$$
\psi_{1}(y)=0, \quad y \in[a, b]
$$

which is obviously not true, because by assumption, $\psi_{1}$ is a non-zero eigenfunction of $H_{0}$ on $[a, b]$. Thus, we arrive at the contradiction, and must put $\mathbf{v}=\mathbf{0}$. This proves that the $B(x)$-matrix does not have zero-eigenvalues that can cause problems in our algorithm.

\section{Appendix C: Proof of Eq. (14)}

Using equations $(7)$ and 13 we write the elements of the $B(x)$-matrix as

$$
\begin{aligned}
{[B(x)]_{i, j} } & =\sum_{n=1}^{N_{b}} \sum_{m=1}^{N_{b}} C_{i, m} C_{j, n} \frac{2}{L} \int_{0}^{x} \mathrm{~d} z \sin \left(\frac{m \pi z}{L}\right) \sin \left(\frac{n \pi z}{L}\right) \\
& =\sum_{n=1}^{N_{b}} \sum_{m=1}^{N_{b}} C_{i, m} C_{j, n} \frac{1}{L} \int_{0}^{x} \mathrm{~d} z\left[\cos \left((m-n) \frac{\pi z}{L}\right)-\cos \left((m+n) \frac{\pi z}{L}\right)\right] .
\end{aligned}
$$

The integral yields $L[f(x)]_{m, n}$, where the function $f(x)$ is defined in Eq. 15, therefore,

$$
\begin{aligned}
{[B(x)]_{i, j} } & =\sum_{n=1}^{N_{b}} \sum_{m=1}^{N_{b}} C_{i, m}[f(x)]_{m, n} C_{j, n} \\
& =\left(C f(x) C^{\mathrm{T}}\right)_{i, j}
\end{aligned}
$$

which is what we wanted to show.

\section{Appendix D: Scaling of the coefficients}

In this section we prove the scaling of coefficients in the case of homogeneous potentials $(26)$. For this we consider a homogeneous potential, i.e., there exist a point $a \in(0, L)$ and a real number $s$ such that for any $k \in \mathbb{R}$ the following holds

$$
V(k(x-a))=k^{s} V(x-a)
$$


Without loss of generality, we assume that $a=0$. This leads to the dimensionless potential

$$
\tilde{V}(\tilde{x})=2 \ell^{2} \ell^{s} V(\tilde{x})=2 \ell^{s+2} V(\tilde{x})
$$

We see that if we scale the potential and $\ell$ at the same time as follows

$$
V(x) \rightarrow \gamma V(x), \quad \ell \rightarrow \ell^{\prime}=\ell \gamma^{-\frac{1}{s+2}},
$$

the dimensionless potential will not change, and, therefore, the dimensionless geometric coefficients returned by CONAN should be the same, i.e.,

$$
\tilde{\alpha}_{k}\left[2 \ell^{2} V(x / \ell)\right]=\tilde{\alpha}_{k}\left[2 \ell^{\prime 2} \gamma V\left(x / \ell^{\prime}\right)\right]
$$

which using Eq. 17) leads to the scaling presented in Eq. (26)

$$
\alpha_{k}[\gamma V]=\gamma^{\frac{3}{s+2}} \alpha_{k}[V]
$$

[1] Y. V. Nazarov and Y. M. Blanter: Quantum Transport Introduction to Nanoscience, (Cambridge University Press, New York, 2009).

[2] V. V. Deshpande, M. Bockrath, L. I. Glazman, and A. Yacoby, Nature 464, 209 (2010).

[3] A. Bezryadin: Superconductivity in Nanowires, (WILEYVCH Verlag, Weinheim, Germany, 2013).

[4] F. Altomare and A. M. Chang: One-Dimensional Superconductivity in Nanowires, (WILEY-VCH Verlag, Weinheim, Germany, 2013).

[5] S. J. Blundell and F. L. Pratt, Journal of Physics: Condensed Matter 16, R771 (2004).

[6] M. Mourigal et al., Nature Phys. 9, 435 (2013).

[7] S. Sahling et al., Nature Phys. 11, 255 (2015).

[8] T. Giamarchi: Quantum Physics in One Dimension (Oxford University Press, Oxford, 2003).

[9] M. Takahashi: Thermodynamics of One-Dimensional Solvable Models (Cambridge University Press, Cambridge, 1999)

[10] U. Schollwöck, Rev. Mod. Phys. 77, 259 (2005).

[11] U. Schollwöck, Ann. Phys. 326, 96 (2011).

[12] M. Lewenstein et al., Adv. Phys. 56, 243 (2007).

[13] I. Bloch, J. Dalibard, and W. Zwerger, Rev. Mod. Phys. 80, 885 (2008).

[14] T. Esslinger, Ann. Rev. Cond. Matter Phys. 1, 129 (2010).

[15] M. A. Baranov, M. Dalmonte, G. Pupillo, and P. Zoller, Chem. Rev. 112, 5012 (2012).

[16] N. T. Zinner and A. S. Jensen, J. Phys. G:Nucl. Part. Phys. 40, 053101 (2013).

[17] L. W. Tonks, Phys. Rev. 50, 955 (1936).

[18] M. D. Girardeau, J. Math. Phys. 1, 516 (1960).

[19] M. Olshanii, Phys. Rev. Lett. 81, 938 (1998).

[20] B. Paredes et al., Nature 429, 277 (2004).

[21] T. Kinoshita, T. Wenger, and D. S. Weiss, Science 305, 1125 (2004).

[22] T. Kinoshita, T. Wenger, and D. S. Weiss, Nature 440, 900 (2006).

[23] E. Haller et al., Science 325, 1224 (2009).

[24] E. Haller et al., Nature 466, 597 (2010).
[25] G. Pagano et al., Nature Phys. 10, 198 (2014).

[26] F. Serwane et al., Science 332, 336 (2011).

[27] G. Zürn et al., Phys. Rev. Lett. 108, 075303 (2012).

[28] A. Wenz et al., Science 342, 457 (2013).

[29] S. Murmann et al., Phys. Rev. Lett. 114, 080402 (2015).

[30] S. Murmann et al., Phys. Rev. Lett. 115, 215301 (2015).

[31] M. D. Girardeau and M. Olshanii, Phys. Rev. A 70, 023608 (2004).

[32] M. D. Girardeau, Phys. Rev. Lett. 97, 210401 (2006).

[33] F. Deuretzbacher et al., Phys. Rev. Lett. 100, 160405 (2008).

[34] L. Guan, S. Chen, Y. Wang, and Z.-Q. Ma, Phys. Rev. Lett. 102, 160402 (2009).

[35] M. Ogata and H. Shiba, Phys. Rev. B 41, 2326 (1990).

[36] S. E. Gharashi and D. Blume, Phys. Rev. Lett. 111, 045302 (2013).

[37] E. J. Lindgren et al., New J. Phys. 16063003 (2014).

[38] A. G. Volosniev et al., Nature Commun. 5, 5300 (2014).

[39] F. Deuretzbacher et al., Phys. Rev. A 90, 013611 (2014).

[40] A. G. Volosniev et al., Phys. Rev. A 91, 023620 (2015).

[41] J. Levinsen et al. Science Advances, 1, e1500197 (2015).

[42] X. Cui and T.-L. Ho, Phys. Rev. A 89, 023611 (2014).

[43] X. Cui and T.-L. Ho, Phys. Rev. A 89, 013629 (2014)

[44] L. Yang, L. Guan, and H. Pu, Phys. Rev. A 91, 043634 (2015).

[45] L. Yang and X. Cui, Phys. Rev. A 93, 013617 (2016).

[46] O. V. Marchukov et al., arXiv:1508.07164 (2015).

[47] N. J. S. Loft et al., arXiv:1508.05917 (2015).

[48] P. Massignan, J. Levinsen, and M. M. Parish, Phys. Rev. Lett. 115, 247202 (2015).

[49] L. Yang and H. Pu, arXiv:1601.02556 (2016).

[50] The CONAN souce code and precomplied versions can be found at http://phys.au.dk/forskning/forskningsomraader/amo/fervbody-physics-in-a-many-body-world/conan/

[51] N. J. S. Loft et al., New J. Phys. 18, 045011 (2016).

[52] F. Deuretzbacher et al., arXiv:1602.06816 (2016). 